\title{
A Visual Decision-Support System using Fingerprint Matrices applied to Cyclical Spatio-Temporal Data from Motorsports
}

\author{
Chris Street \\ University of Regina \\ chris.street@uregina.ca
}

\author{
Orland Hoeber \\ University of Regina \\ orland.hoeber@uregina.ca
}

\begin{abstract}
Visualizing cyclical spatio-temporal data is an important part of understanding how and why objects move in the context of motorsports, which is critical feedback for drivers to improve their performance. Current methods have problems such as occlusion and loss of context which significantly limit our ability to see and understand vehicle data. Here we demonstrate how the fingerprint matrix method (which is normally used in lexical analysis) can be applied in vehicle motion analysis to overcome these two problems. Compared to traditional methods using traction circle scatterplot displays of acceleration force data from a race car, our prototype design allows decision makers to see individual datapoints in a more concise display. We show that informative but previously-hidden anomalies and patterns become more easily recognized in the data. Our design generalizes to other cyclical spatio-temporal visualization problems involving transportation, medicine, and the natural world.
\end{abstract}

\section{INTRODUCTION}

A critical step in vehicle-centered data analytics involves collecting and analyzing information about the car, track, and driver while the vehicle is in motion. Data about the dynamic handling characteristics of a race car provide critical information for drivers and race crews to understand and improve how a car reacts to mechanical changes meant to make the car faster [1][2]. So much engineering and physiological information is available to race teams that visualizing this information has become a critical part to understanding how a car [3] and driver [4] are reacting to stresses. In motorcar racing, the analysis process involves data about objects that move in recurring spatio-temporal cycles (i.e. laps on a track) [5].

Interpreting spatio-temporal data visualizations is an example of a larger research problem in vehicle telemetry analytics and the development of multi-sensor autonomous vehicles. Research on data visualization techniques for developing multi-sensor vehicles involves both autonomous vehicle operation systems [6] [7] and human analyst systems [8] [9] where one goal is to minimize the visual interference that occurs when working with real-time multi-sensor data. In the context of human analyst systems there are two forms of visual interference, occlusion [10] [11] and loss of context when zooming in to reveal detail [12].

This paper presents a design study and prototype implementation of a decision-support visualization using the fingerprint matrix method [13] to show cyclical spatiotemporal data in a way that avoids the problems of occlusion and loss of context for human analysts. This is a novel application of the fingerprint matrix method; to our knowledge, it is the first time it has been used to support the analysis of cyclical spatio-temporal data. In this research, motorsports serves as a representative example of a data-rich domain in which cyclical spatio-temporal data is prevalent, enabling us to experiment with and reason about different approaches for visualizing and analyzing such data.

The remainder of this paper is organized as follows. The next section explains the relevant prior work in spatiotemporal visualization as well as a brief description of the fingerprint matrix design and its application in computational literary analysis. The third section presents the design thinking approach [14] for functional analysis and visualization development. Following a design thinking approach [15], we then present and analyze the design study. We conclude with a discussion on the generalizability of the design and its limitations.

\section{Prior WORK}

Spatio-temporal data includes features that relate to both space and time, meaning that it is generally about moving objects [5]. Visualizing this type of data involves combining spatial information about physical location with temporal information about when each data point came into existence. The spatial representation can be a physical map, a diagram of the human body, or locations on some physical object. Temporal order can be chronological (e.g. clock time), relative (e.g. this happened, then three seconds later this happened), or sequential (e.g. this happened first, followed by something else) [16]. Interactive visualization tools and animation are often added to spatial displays such as a road map or diagram of the human circulatory system, to provide 
analysts with the ability to view the temporal aspects of the data with a goal of searching for or confirming trends, rates of change, or contact [17]. Visualizing spatio-temporal data involves organizing a careful symphony of three streams of information (a position in space, a point in time, and a measure of something happening at that space and time) and then displaying that data point in a meaningful way to an analyst [18].

Cyclical spatio-temporal refers to data that involves objects moving in recurring patterns. This context generalizes to an analysis problem that involves an object (e.g. a race car), a cyclical spatio-temporal activity (e.g. driving laps around a track), and information of interest to an analyst (e.g. peak acceleration forces on the car). Visualizing this type of data is an important tool in a wide variety of research domains, including human, animal, engineering, logistics, and social science research [19].

When the object of interest is circling through a bounded space such as a race track, the individual data points begin to overlay in visual space and become difficult to perceive from one another. Prior designs have attempted to solve some of these problems with distortion-based designs such as the Hyperbolic Browser [20] and Fisheye Views [21] or projection-based designs such as the Magic Eye [22]. In this paper we use fingerprint matrices, a non-distortion, nonprojection approach, to provide a straightforward and concise way to visualize cyclical spatio-temporal data.

Fingerprint matrices (FM) are an overview visualization that are meant to be matched with a detail visualization through multiple coordinated views [23]. An FM aggregates key data into a single value that is represented with a colour intensity that helps users identify and analyze important relationships in a dataset [24]. The design is typically used to visualize characteristics in two types of datasets: bodies of text (literary fingerprinting) and musical compositions (harmonic fingerprinting).

Literary fingerprinting refers to the visualization of textual characteristics in order to compare writing styles of authors [25] or the content of a text to identify how themes evolve over time [26]. Prior work has shown that the FM is effective for identifying and comparing quantitative characteristics of text [27]-[29].

Musical analysis also uses the FM design. Harmonic fingerprinting [30] displays rhythm and harmony information for viewers in the same way as text analysts use an FM. Prior research has also used the FM as the overview component in an overview+detail design for visualizing harmony and melody for individual instruments in a musical score [31]. These studies show that the fingerprint matrix can be used for visualizing recurring patterns in data that have a spatial component (e.g. relative location of words/themes in a text) and a temporal component (e.g. ordering of notes in a score).

\section{Design Thinking ApProach}

Race teams are responsible for creating a competitive race car by managing the car, the driver, and the race track characteristics in ways that the car is always performing at the limits of physics. One of the most important analytical elements are the acceleration forces ( $g$-forces) acting on a car as it races around a track. Data is collected by an accelerometer telemetry device inside the car that records forces as the car travels on the race track. Straight-line and lateral acceleration forces are exerted as the car accelerates, brakes, and turns, all acting in opposition to the friction force between the car's tires and the surface of the race track. If the g-forces exceed the tires' friction force with the race track then the tires will lose grip, spin, and possibly cause the car to go out of control. A common visualization approach for showing the forces acting on a race car is a traction circle (TC) which visualizes the straight-line and lateral forces on a car [32] (See Figure 1a).

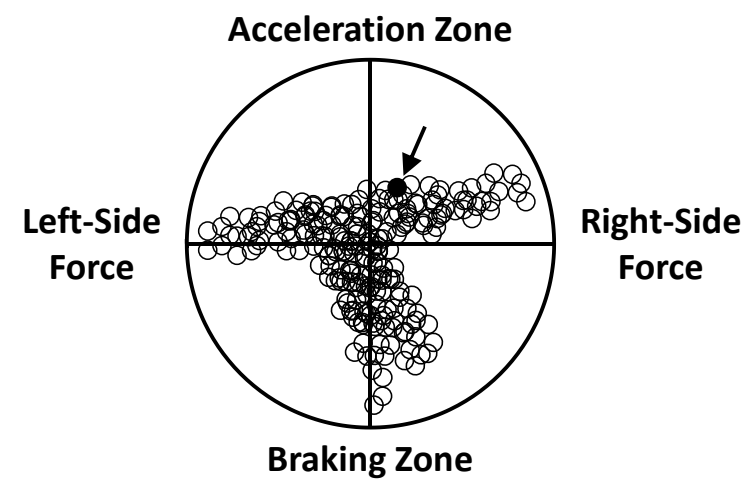

(a) Occlusion when dataset is plotted, arrow indicates current datapoint of interest

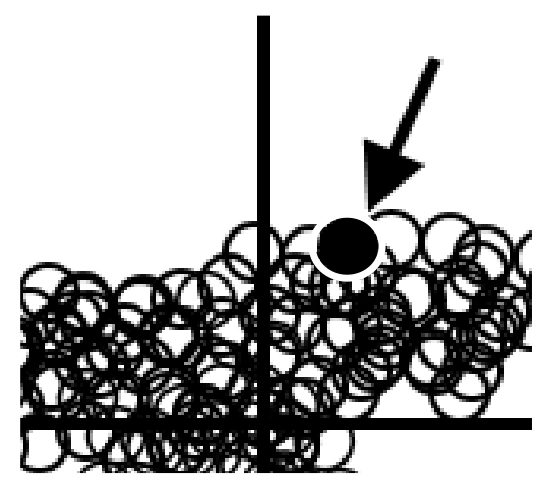

(b) Loss of context when scatterplot is zoomed in to better see individual datapoints, can no longer see the pattern in the rest of the data

Figure 1. Occlusion and context loss [33] 
A TC is an x-y scatterplot where each data point contains an $\mathrm{x}$ (left or right lateral force) and $\mathrm{y}$ (accelerating or braking straight-line force) coordinate.

Traction circle visualizations are subject to occlusion problems when a series of overlaying points obscure individual datapoints (see Figure 1b). They are also subject to context loss when zooming in [17]. Human cognition is degraded when something goes out of view [27] so in cases such as racing where the total force picture over periods of time must be perceived and interpreted, loss of context makes it more difficult for race teams to analyze and identify patterns and trends. A better visualization approach would involve displaying the information in a way that minimizes or avoids both problems.

The analyst in this context is a race team consisting of a mechanic crew and a driver, whose goals are to adjust and drive the car to its maximum capacity, respectively. The analyst's task is to find patterns in the TC scatterplot that indicate known operational patterns such as smooth driving through a sharp turn. Trained and experienced mechanics, engineers, and drivers can watch for key locations on a track, such as entries into corners, and watch how the forces on the car change, how much they change, and if the changes are consistent at the same places in each lap. In much the same way that a statistician examines a scatterplot to see if there are patterns indicating linear, quadratic, or exponential relationships in the data, race teams examine a TC scatterplot to see if there are indications of smooth corner entries and exits or unplanned tire spins [34]. Figure 1 shows how occlusion and context loss makes visual analysis difficult, which provided motivation for designing and developing an approach that avoids this problem.

Our goal in this research is to visualize acceleration force data that varies in intensity. In motorcar racing, drivers have a 'traction budget' [35] which represents the maximum acceleration force the car can experience before the tires lose grip with the track surface and the driver loses control of the car. As the name suggests, the 'budget' does not care what direction the acceleration force is coming from, only whether the total force vector is less than the maximum peak grip value. We propose that the optimal design for the analyst is to visualize the peak acceleration force data of a car as a single continuous value. The task requirements for the visualization are therefore (1) perceive the peak force acting on the car throughout a race; (2) identify known temporal patterns and trends in the force data; and (3), map these patterns and trends spatially and temporally to the race track in order to make sense of what is happening to the car and driver.

In order for the analyst to make sense of the force acting on the car we need to allow them to be able to see at least three coordinated views of the data: the peak-force values at specific track positions for a complete race (overview); the two-dimensional point-force on the car (detail); and the spatial position of the car on the track (detail). Visualizing the point-force data and the spatial position for a car can be represented by a standard traction circle scatterplot and a race track wireframe image, respectively. Our novel contribution in this work is to propose that the comprehensive peak-force information can be represented by a fingerprint matrix. Following the Gestalt Principle of Similarity [36], the mark on the track, traction circle, and FM have visually identical attributes for colour, outline, and motion to encourage the interpretation that they are all related.

Figure 2 shows a screen shot of the visualization prototype. The data for this prototype includes seven attributes; speed, engine RPM, latitude and longitude GPS coordinates, $\mathrm{x}$ - and $\mathrm{y}$-force acceleration figures, and a timestamp. A synthetic dataset based on real-world motorsports characteristics was created to illustrate the prototype.

The right-hand top panel shows acceleration force data in an overview (fingerprint matrix) and detail (the more familiar traction circle) manner. The display to the left of the FM shows the car as a mark overlaid on a track. The car is represented as a green circle (track and TC) or rectangle (FM) with a black outline to represent to the analyst that the three marks are linked in order to support contextual awareness at each point in time.

As an information overview, the FM is composed of rows of individual cells. Each row represents one lap of the track and the rows are arranged temporally from top to bottom to represent first lap to last lap. Each column (cell) in the row represents one acceleration force data point. The cells are arranged temporally from left to right to represent the first acceleration force data point for the lap to the last data point for the lap. Each cell is mapped to a segment of the track to represent the force on the car at that point. In this prototype there are 60 cells mapped to 60 evenly-divided physical segments of the track. The number of cells could be changed or created dynamically by design. An algorithm reads the data for a row/lap and uses the GPS coordinates to segment the data into an array that contains force readings for each segment. The $\mathrm{x}$ - and $\mathrm{y}$-force values are used to calculate the total force vector magnitude for each datapoint in a segment and the maximum (peak) force value is used to represent total force for that cell/segment. Once each segment is represented by a peak force magnitude, the values are normalized to a $0-1$ scale and the saturation value for each cell is set to the normalized value. This process creates cell colours that vary in intensity from low to high to correspond to total force values that similarly vary from low to high. 


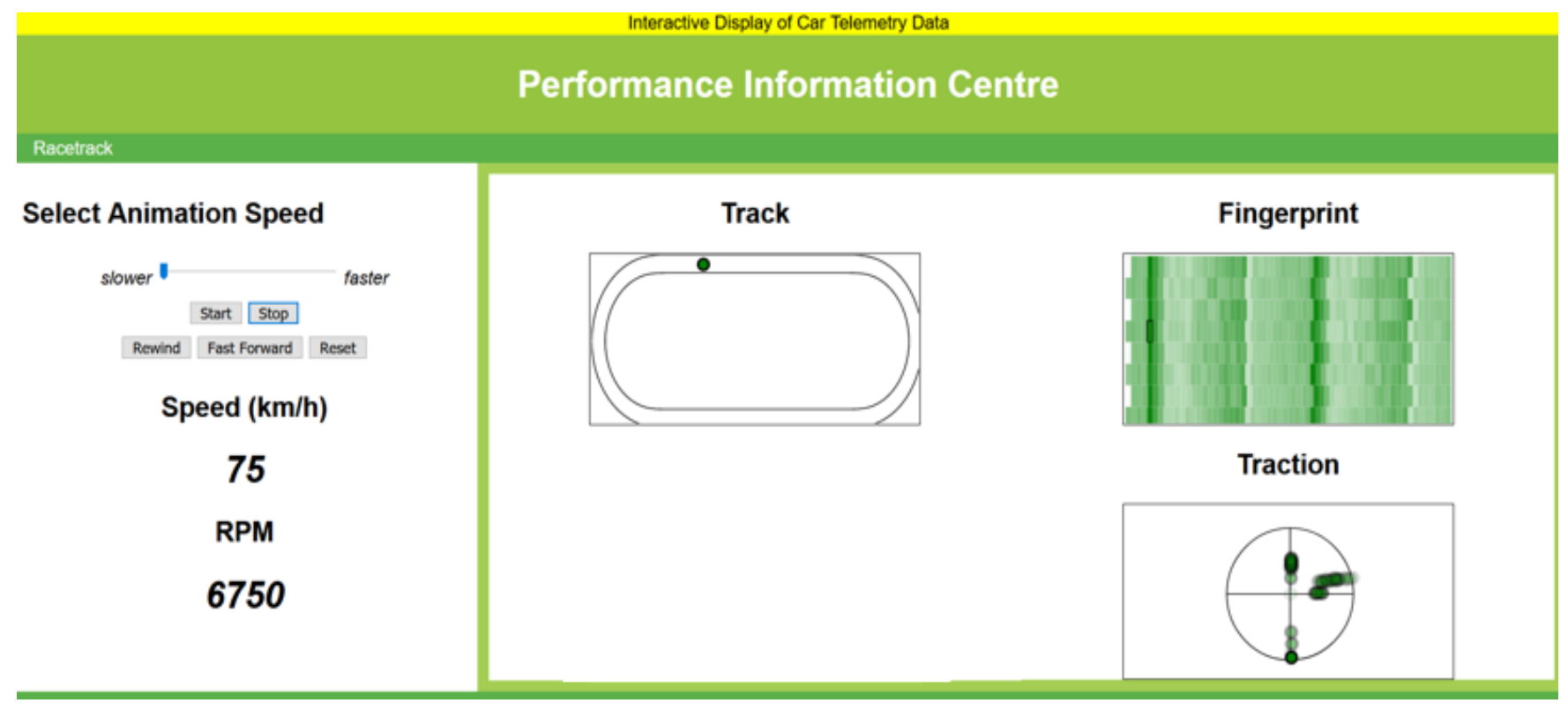

Figure 2. Prototype interface

This use of colour intensity to represent the numerical value of the peak force has a theoretical basis in the work by Mackinlay on matching visual variables to data types [37] representing the lightness/darkness channel in the Opponent Process Theory of Colour [38].

The traction circle detail view is composed of an $x-y$ plot with lateral (side-to-side) force displayed on the $\mathrm{x}$-axis and acceleration/braking force displayed on the $y$-axis. The $x-y$ origin represents zero force, while left-side lateral and braking forces are negative numbers and right-side lateral and acceleration forces are positive. Each mark in the plot represents one force data point. Each point is initially shown as a fully-opaque green mark when first displayed and then faded to $15 \%$ transparency in order to allow it to be seen if other data points later overlap it. When there are a small number of data points, this partially addresses the issue of occlusion, but does not scale well.

The race track detail view is composed of a wireframe outline of the inner and outer boundaries of the track and are drawn using static latitude/longitude coordinates. The race car mark that travels around the wireframe track is positioned using a mapping function that transforms the latitude/longitude coordinates recorded with each data collection point into screen coordinates. Mark animation is done using a visual transition function that redraws the mark sequentially from one position to the next in order to create a visually pleasing motion.

The overview and detail views are coordinated in three ways. The FM and race track views are coordinated spatially by mapping one lap of the track to one row of the matrix. The start/finish line of the track corresponds to the first and last cells in a row, a third of the way through the track is also a third of the way through the row, and so on. These two views are also coordinated temporally because the tempo of the mark on the race track view moves at the same tempo as the FM mark. Finally, the FM and traction circle are coordinated such that the TC shows the $\mathrm{X}$ and $\mathrm{Y}$ axis forces on the car at a given point while the FM shows the magnitude of the peak or maximum force vector of that same point. Taken together, the FM shows the peak force relevant for understanding the traction budget while the TC breaks down that peak to the $\mathrm{x}$ and $\mathrm{y}$ components relevant for understanding if traction is being lost to excessive braking, turning, or accelerating. Adding the race track view, the analyst can also see the track position where this is occurring.

When used in replay mode, interaction controls are provided to change the animation speed and behaviour. Start/Stop controls are provided to freeze-frame and resume animation. Fast forward, rewind, and reset controls are provided to easily replay certain portions of the track, jump to specified places, or restart the animation. Two modes of operation exist, replay and real-time. In replay mode analysts are expected to watch the animation and evaluate the changes in force acting on the car as the laps proceed. In replay mode the full race would be represented immediately in the FM.

This prototype was initially designed for replay mode but could be extended to real-time in a design iteration to add a redraw() function that is initiated by the car crossing the start/finish line. This function would redraw the view with an additional row each time a new lap is recorded, continuing 
to add rows until the race is complete. Figure 2 shows replay mode.

This design was implemented using JavaScript, the D3.js libraries, and PHP. XAMPP was used for the local development environment (Apache 2.4.43, MariaDB 10.4.13, PHP 7.2.32). JavaScript libraries were not available for track animation, traction circles, or the fingerprint matrix so these code libraries were developed new for this work.

\section{Design Critique \& Scenario Analysis}

Two important questions for evaluating whether this design improves upon current practice are: (1) how is the FM visualization different from the TC?, and (2) does the design create a more useful visualization for the analyst? Overall, Figure 3 shows that the FM's novel design displays all the same data as the Traction panel but does not occlude datapoints. In addition, there is no need to zoom in to see detail with the FM design because the rectangular marks representing each datapoint occupy their own nonoverlapping space and can still be seen at full scale. Two scenarios are presented to illustrate how the fingerprinting visualization is different and more useful to an analyst.

Figure 3(a) shows two points on the racetrack, a and b, that represent a vehicle entering turns 1 and 3 respectively. These points are represented simultaneously in three coordinated views, allowing an analyst to choose which to observe depending on the type of information they are seeking. The fingerprint matrix provides an overview of the forces that were exerted on the car over the race, enabling high-level patterns to be observed. Detailed information about the specific direction and magnitude of forces are shown on the traction circle, enabling a comparison to previous data. Where the data was measured is shown on the track visualization, adding context to the analysis.

Vehicle motion and forces in corners are critical for analysts to visualize because they represent the point where a vehicle is most vulnerable to being passed by another car, hence wanting the car to move as fast and smooth as possible around turns. Over the course of a race the vehicle analysts would collect vehicle telemetry data such as forces on the car for each lap and visualize the data after the race for analysis purposes. Two scenarios illustrate how the fingerprinting design can be more useful to analysts in understanding their data.

The first scenario involves the driver and crew reviewing the data for anomaly detection such as determining if the vehicle operated within expected limits. Using the traction circle an analyst could compare the $(x, y)$ datapoints that correspond to certain positions of interest on the racetrack.

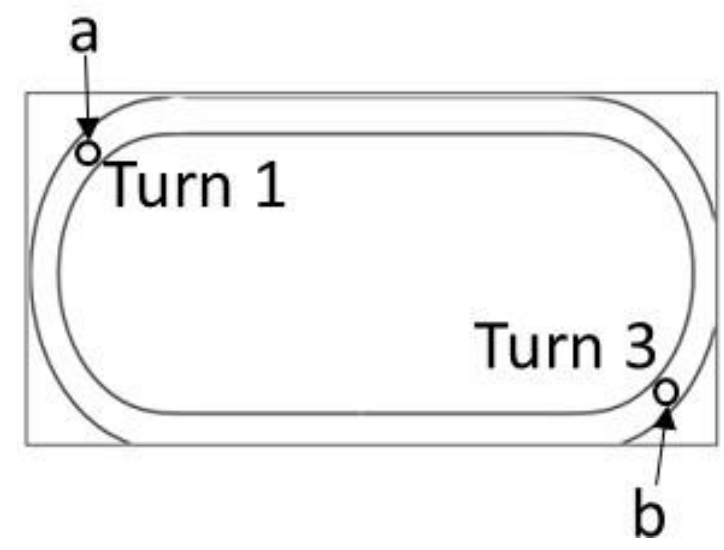

(a) Track Trace

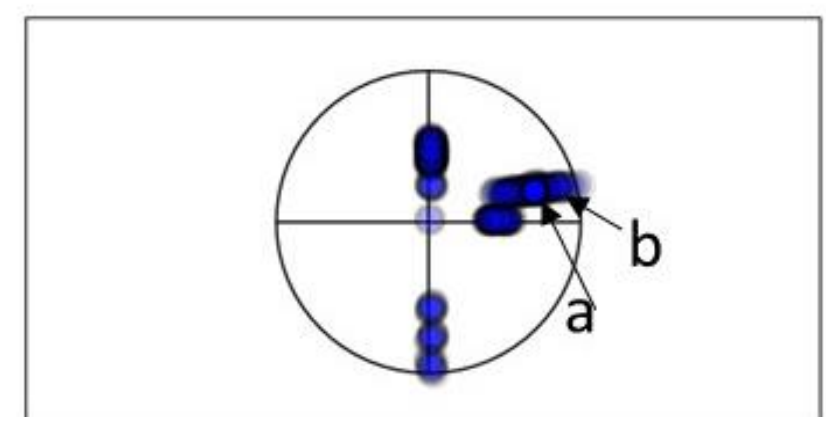

(b) Traction Circle Visualization

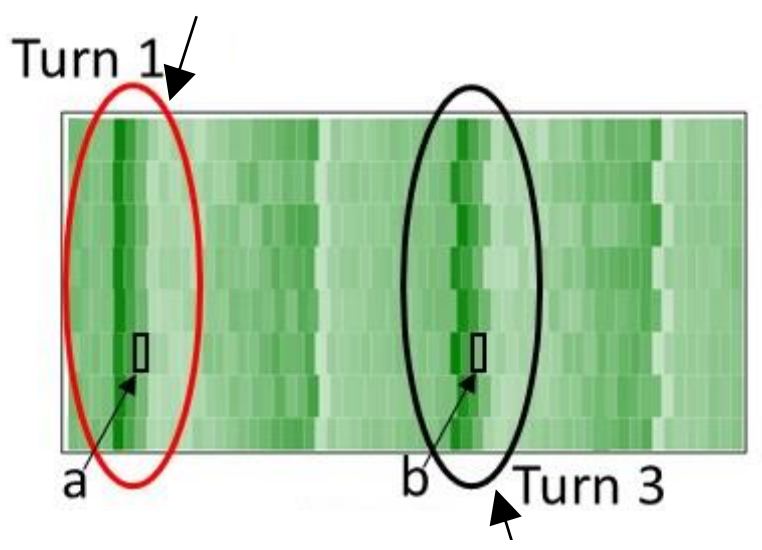

(c) Fingerprint Matrix Visualization

Figure 3. Comparing FM and TC

However, as Figure 3(b) shows, because the forces are relatively similar at the same position on a track over 
multiple laps, the datapoints on the traction circle are heavily overlaid and it's not possible to see critical differences.

Looking instead to the fingerprint matrix visual however we can see a different view of this same data.

When we map darker cell colors to higher force levels on the car, in Figure 3(c) we see first that the darker cells are starting and ending in cell 5 in each row (lap) which is expected as the car is being 'loaded up' with force at the same point in each lap. However, we see at the other end of the row in the "Turn 3" portion indicated in the FM that the cell colours change in a different way than in the "Turn 1" portion. We can therefore detect a visual anomaly between the forces in turns 1 and 3, possibly indicating that either the car or the driver is doing something different in one region of the track than the other. While this difference is readily apparent in the fingerprint matrix, it is not visible in the traction circle.

A second scenario involves pattern recognition tasks such as determining whether a critical point or anomaly repeats itself in recognizable patterns. Continuing the 'turns 1 \& 3' example, an analyst might review the data to determine if the vehicle and driver are operating in expected ways throughout a race. Smooth and consistent g-force loading typically results in a faster lap time so analysts are looking for patterns in their data that suggest minimal variance from one lap to the next. Reviewing Figure 3(b), it is difficult to see recurring patterns at any detail, again because the datapoints are severely superimposed. Zooming in on specific regions does not solve the problem because the adjacent points needed to perceive lap-to-lap differences quickly go offscreen.

The FM in Figure 3(c) again shows a better picture for analysts. The bottom panel shows a recurring pattern of irregular driving at the turn 3 end of the track that does not occur at the turn 1 end of the track. With this visualization, an analyst can immediately discern that, in this case, force changes are smooth and consistent in turn 1 (expectations met) but are neither smooth nor consistent in turn 3 (abnormal pattern detected). Different reasons exist to explain why a pattern like this might occur, but most significantly the matrix approach allows an analyst to see the anomaly in the first place. This design encourages comparison and exploration of the data by identifying where and when things are unexpected and supporting inspection of this data to try to reveal the underlying circumstances.

\section{Generalizability, Limitations, \& Future Work}

The design for this prototype generalizes to contexts where an object of interest moves spatially and temporally within a bounded space, and where the analyst wants to see both overview and detail of that object's characteristics free of

Table1. Cyclical geo-temporal analysis contexts

\begin{tabular}{|l|l|}
\hline $\begin{array}{l}\text { Race Car } \\
{[1]}\end{array}$ & $\begin{array}{l}\text { Are the forces on a race car during laps } \\
\text { in a race changing in predictable ways? }\end{array}$ \\
\hline $\begin{array}{l}\text { Animals } \\
{[39]}\end{array}$ & $\begin{array}{l}\text { What food source is being used each } \\
\text { time they follow seasonal migration } \\
\text { routes? }\end{array}$ \\
\hline $\begin{array}{l}\text { Fishing } \\
{[40]}\end{array}$ & $\begin{array}{l}\text { Do fishing vessels show anomalous } \\
\text { behaviour as they cycle between port and } \\
\text { fishing grounds? }\end{array}$ \\
\hline $\begin{array}{l}\text { Delivery } \\
\text { vehicles } \\
{[41]}\end{array}$ & $\begin{array}{l}\text { How does fuel economy change as the } \\
\text { delivery trucks become empty at } \\
\text { different times of the day? }\end{array}$ \\
\hline $\begin{array}{l}\text { Medicine } \\
{[42]}\end{array}$ & $\begin{array}{l}\text { How does the absorption of imaging dye } \\
\text { change as it cycles through the major } \\
\text { arteries of the circulatory system? }\end{array}$ \\
\hline
\end{tabular}

visual interference. The motivation for this research was to develop better human-centered visualization systems of moving vehicle data in racing or autonomous vehicle contexts. Table 1 shows additional examples of where analysts could benefit from seeing the overview+detail coordinated view of cyclical spatio-temporal data.

Consider the avian example where food sources are being visualized during bird migration [39]. The Fingerprint Matrix design (paired with a geographic map instead of a racetrack) would allow for the display of food sources encountered over multiple migration cycles and show, perhaps, that food sources are changing from one migration cycle (one matrix row) to the next. This information would not be as clear if overlaid on a map, particularly when migration routes are highly consistent from one year to the next. In this situation the migration map would experience the same occlusion and context loss problems as the traction circle in the racing context.

As a concept prototype this design has several limitations that should be acknowledged. First, the underlying data was synthesized in order to operate the functionality of the interface. Care was taken to create the data using physics equations relating speed, mass, travel, and acceleration force, however using a synthetic dataset is not the same as capturing live data. We are currently developing a carmounted telemetry device for collecting force and position data during actual races. 
A second limitation is that the functional evaluation in this paper does not address whether the data visualization actually assists crews or drivers gaining faster lap times. User evaluation testing is needed to validate that analysts are in fact more effective at perceiving and interpreting acceleration force data and can translate that understanding into the practical outcome of going faster. In combination with telemetry device development we are planning user evaluation studies to examine how well this design works for anomaly detection and pattern recognition using real data. Our ultimate research goal is to develop a visual representation of spatio-temporal data for real-time analysis while the events of interest are occurring.

Future work should also include the development of new functionality that allows a user to perform spatial analysis tasks such as being able to select elements in the fingerprint matrix and have information about what was happening to the car and on the track at that time and location. This style of interactivity should also go in the other direction and allow users to select positions on the track and have those positions highlighted in the FM. In both cases, the selection of multiple data points could support comparative analysis of the data. With such features, users would be able to perform 'what if' analyses by selecting high-force areas of interest in the FM to see where on the track that cars are experiencing high g-loads, or selecting different start-stop positions on the track to find areas where cars are going the fastest or the slowest.

To conclude, our design intent was to bridge an existing shortcoming in how data is visualized in motorcar racing. Our novel design used the fingerprint matrix that is normally used for literary and musical analysis and extended this approach into the domain of analyzing cyclical spatiotemporal data. This is our first step in applying the design into other similar areas of research. We hope that this visualization can be used to improve the presentation of data in better ways.

\section{REFERENCES}

[1] M. Stoll, R. Krüger, T. Ertl, and A. Bruhn, "Racecar Tracking and its Visualization Using Sparse Data," in Proceedings - Workshop on Sports Data Visualization, 2013, pp. 1-6, [Online]. Available: http://workshop.sportvis.com/papers/stoll.pdf.

[2] T. Lampprecht, D. Salb, M. Mauser, H. Van De Wetering, M. Burch, and U. Kloos, "Visual Analysis of Formula One Races," in Proceedings of the International Conference on Information Visualisation, 2019, pp. 9499, doi: 10.1109/IV.2019.00025.
[3] O. Ehirim, "Optimal diffuser design for Formula SAE race car using an innovative geometry buildup and CFD simulation setup with on-track testing correlation," $S A E$ Tech. Pap. No. 2012-01-1169, 2012, doi: 10.4271/201201-1169.

[4] E. S. Potkanowicz and R. W. Mendel, "The case for driver science in motorsport: A review and recommendations," Sport. Med., vol. 43, no. 7, pp. 565574, 2013, doi: 10.1007/s40279-013-0040-2.

[5] M. Erwig, R. H. Güting, M. Schneider, and M. Vazirgiannis, "Spatio-temporal data types: An approach to modeling and querying moving objects in databases," Geoinformatica, vol. 3, no. 3, pp. 269-296, 1999, doi: 10.1023/A:1009805532638.

[6] Q. Alrousan, H. Alzu'bi, A. Pfeil, and T. Tasky, "Autonomous Vehicle Multi-Sensors Localization in Unstructured Environment," SAE Tech. Pap., 2020, doi: 10.4271/2020-01-1029.

[7] T. A. Siddiqui, R. Madhok, and M. O'Toole, "An extensible multi-sensor fusion framework for 3D imaging," in IEEE Computer Society Conference on Computer Vision and Pattern Recognition Workshops, 2020, pp. 1008-1009, doi:

10.1109/CVPRW50498.2020.00512.

[8] D. Saffo, A. Leventidis, T. Jain, M. A. Borkin, and C. Dunne, "Data Comets: Designing a Visualization Tool for Analyzing Autonomous Aerial Vehicle Logs with Grounded Evaluation," Comput. Graph. Forum, 2020, doi: 10.1111/cgf.13994.

[9] V. Lizenberg, B. Büchs, S. Knapp, R. Mannale, and F. Köster, "Graphical data visualization for vehicular communication systems in real and virtual test environments," in AmE 2020: Automotive meets Electronics - 11. GMM-Fachtagung, 2020, pp. 1-6.

[10] D. A. Keim, M. C. Hao, U. Dayal, H. Janetzko, and P. Bak, "Generalized scatter plots," Inf. Vis., vol. 9, no. 4, pp. 301-311, 2010, doi: 10.1057/ivs.2009.34.

[11] A. Prouzeau, M. Cordeil, C. Robin, B. Ens, B. H. Thomas, and T. Dwyer, "Scaptics and highlight-planes: Immersive interaction techniques for finding occluded features in 3D scatterplots," in Conference on Human Factors in Computing Systems - Proceedings, 2019, pp. 1-6, doi: 10.1145/3290605.3300555.

[12] I. Herman, G. Melançon, and M. S. Marshall, "Graph visualization and navigation in information visualization: a survey," IEEE Trans. Vis. Comput. Graph., vol. 6, no. 1, pp. 24-43, 2000, doi: 10.1109/2945.841119.

[13] D. A. Keim and D. Oelke, "Literature fingerprinting: A new method for visual literary analysis," in Proceedings 2007 IEEE Symposium on Visual Analytics Science and Technology, 2007, pp. 115-122, doi: 10.1109/VAST.2007.4389004.

[14] M. Meinel, T. T. Eismann, C. V. Baccarella, S. K. Fixson, and K. I. Voigt, "Does applying design thinking result in better new product concepts than a traditional 
innovation approach? An experimental comparison study," Eur. Manag. J., vol. 38, no. 4, pp. 34-41, 2020, doi: 10.1016/j.emj.2020.02.002.

[15] A. Ramaprasad and T. Syn, "Design thinking and evaluation using an ontology," in European Design Science Symposium, 2013, vol. 63-74, doi: 10.1007/9783-319-13936-4_6.

[16] M. Brehmer, B. Lee, B. Bach, N. H. Riche, and T. Munzner, "Timelines Revisited: A Design Space and Considerations for Expressive Storytelling," IEEE Trans. Vis. Comput. Graph., vol. 23, no. 9, pp. 2151-2164, 2017, doi: 10.1109/TVCG.2016.2614803.

[17] S. Liu, W. Cui, Y. Wu, and M. Liu, "A survey on information visualization: recent advances and challenges," Vis. Comput., vol. 30, no. 12, pp. 13731393, 2014, doi: 10.1007/s00371-013-0892-3.

[18] Z. Pan, Y. Liang, W. Wang, Y. Yu, Y. Zheng, and J. Zhang, "Urban traffic prediction from spatio-temporal data using deep meta learning," in Proceedings of the ACM SIGKDD International Conference on Knowledge Discovery and Data Mining, 2019, pp. 1720-1730, doi: 10.1145/3292500.3330884.

[19] P. Compieta, S. Di Martino, M. Bertolotto, F. Ferrucci, and T. Kechadi, "Exploratory spatio-temporal data mining and visualization," J. Vis. Lang. Comput., 2007, doi: 10.1016/j.jvlc.2007.02.006.

[20] J. Lamping and R. Rao, "The hyperbolic browser: A focus + context technique for visualizing large hierarchies," J. Vis. Lang. Comput., vol. 7, no. 1, pp. 3355, 1996, doi: 10.1006/jvlc.1996.0003.

[21] T. Hermann, A. Hunt, and J. Neuhoff, Eds., The Sonification Handbook. Berlin: Logos Verlag, 2011.

[22] J. R. Goodall, W. G. Lutters, P. Rheingans, and A. Komlodi, "Preserving the big picture: Visual network traffic analysis with TNV," in IEEE Workshop on Visualization for Computer Security 2005, VizSEC 05, Proceedings, 2005, pp. 47-54, doi: 10.1109/VIZSEC.2005.1532065.

[23] J. C. Roberts, "State of the art: Coordinated \& multiple views in exploratory visualization," in Proceedings Fifth International Conference on Coordinated and Multiple Views in Exploratory Visualization, CMV 2007, 2007, pp. 61-71, doi: 10.1109/CMV.2007.20.

[24] T. Büring, J. Gerken, and H. Reiterer, "Usability of overview-supported zooming on small screens with regard to individual differences in spatial ability," in Proceedings of the Workshop on Advanced Visual Interfaces, 2006, pp. 233-240, doi: $10.1145 / 1133265.1133310$.

[25] C. Kim, U. Hinrichs, S. M. Mohammad, and C. Collins, "Lexichrome: Text construction and lexical discovery with word-color associations using interactive visualization," in DIS 2020 - Proceedings of the 2020 ACM Designing Interactive Systems Conference, 2020, pp. 477-488, doi: 10.1145/3357236.3395503.
[26] F. Wanner, C. Rohrdantz, F. Mansmann, D. Oelke, and D. A. Keim, "Visual sentiment analysis of RSS news feeds featuring the US presidential election in 2008," in Workshop on Visual Interfaces to the Social and the Semantic Web, 2009, pp. 1-8.

[27] D. Oelke, P. Bak, D. A. Keim, M. Last, and G. Danon, "Visual evaluation of text features for document summarization and analysis," in VAST'08 - IEEE Symposium on Visual Analytics Science and Technology, Proceedings, 2008, pp. 75-82, doi: 10.1109/VAST.2008.4677359.

[28] D. Oelke and D. Kokkinakis, "Advanced Visual Analytics Methods for Literature Analysis," in Proceedings of the 6th EACL Workshop on Language Technology for Cultural Heritage, Social Sciences, and Humanities, 2012, pp. 35-44.

[29] M. Baumann et al., "An Interactive Visualization for the Analysis of Annotated Text Variance in the Legendary Der Heiligen Leben, Redaktion.," in LEVIA'19: Leipzig Symposium on Visualization in Applications, 2020, pp. $1-9$.

[30] M. Miller, A. Bonnici, and M. El-Assady, "Augmenting music sheets with harmonic fingerprints," in Proceedings of the ACM Symposium on Document Engineering, DocEng 2019, 2019, pp. 1-10, doi: $10.1145 / 3342558.3345395$

[31] G. D. Cantareira, L. G. Nonato, and F. V. Paulovich, "MoshViz: A Detail+Overview Approach to Visualize Music Elements," IEEE Trans. Multimed., vol. 18, no. 11, pp. 2238-2246, 2016, doi: 10.1109/TMM.2016.2614226.

[32] A. Bonci, R. De Amicis, S. Longhi, and E. Lorenzoni, "On the Traction Control of Single-Track Vehicles in Different Trim Conditions," in 2018 14th IEEE/ASME International Conference on Mechatronic and Embedded Systems and Applications, MESA 2018, 2018, pp. 1-6, doi: 10.1109/MESA.2018.8449200.

[33] T. Quiroga, "How to read a traction circle," Car and Driver, 2010.

https://www.caranddriver.com/features/a15129563/howto-read-a-friction-circle/ (accessed Dec. 11, 2020).

[34] W. C. Mitchell, R. Schroer, and D. B. Grisez, "Driving the traction circle," in SAE Technical Papers, 2004, pp. 1-18, doi: 10.4271/2004-01-3545.

[35] B. Beckman, "The Traction Budget," in The Physics of Racing, Burback CA: NBRC, 1991, pp. 21-26.

[36] K. Koffka, Principles of Gestalt Psychology. London, UK: Routledge, 1935.

[37] J. Mackinlay, "Automating the Design of Graphical Presentations of Relational Information," ACM Trans. Graph., vol. 5, no. 2, 1986, doi: 10.1145/22949.22950.

[38] L. M. Hurvich and D. Jameson, "An opponent-process theory of color vision," Psychol. Rev., vol. 64, no. 6 PART 1, 1957, doi: 10.1037/h0041403. 
[39] N. Ferreira et al., "Birdvis: Visualizing and understanding bird populations," IEEE Trans. Vis. Comput. Graph., vol. 17, no. 12, pp. 2374-2383, 2011, doi: 10.1109/TVCG.2011.176.

[40] O. Hoeber and M. U. Hasan, "A geovisual analytics approach for analyzing event-based geospatial anomalies within movement data," Inf. Vis., vol. 17, no. 2, pp. 91107, 2018, doi: 10.1177/1473871617693040.

[41] C. Fikar, "A decision support system to investigate food losses in e-grocery deliveries," Comput. Ind. Eng., vol. 117, pp. 282-290, 2018, doi: 10.1016/j.cie.2018.02.014.

[42] N. Nishigori et al., "Visualization of lymph/blood flow in laparoscopic colorectal cancer surgery by ICG

fluorescence imaging (Lap-IGFI)," Ann. Surg. Oncol., vol. 23, no. 2, pp. 266-274, 2016. 